\section{Metástasis pancreática como debut de carcinoma papilar de tiroides. Caso clínico y revisión del perfil clínico patológico y molecular}

\author{
THOMAS USLAR ${ }^{1}$, JAVIER CHAHUÁN ${ }^{2}$, ROBERTO OLMOS ${ }^{1,6}$, \\ CAROLINA RODRÍGUEZ ${ }^{3}$, ROCIO ASTUDILLO ${ }^{a}$, \\ PABLO ZOROQUIAIN ${ }^{4}$, ANTONIETA SOLAR ${ }^{4}$, \\ ALBERTO ESPINO ${ }^{2}$, AUGUSTO LEÓN ${ }^{3}$, CARLOS FARDELLA ${ }^{1,5}$, \\ JOSÉ MIGUEL DOMÍNGUEZ ${ }^{1,5}$
}

\section{Papillary thyroid carcinoma presenting with pancreatic metastases. Report of one case}

Pancreatic metastases of papillary thyroid carcinoma (PTC) are exceptional. We report a 80-year-old man consulting for obstructive jaundice and dysphonia. Abdominal ultrasonography showed biliary dilation and abdominal magnetic resonance imaging (MRI) showed a pancreatic head mass of $36 \mathrm{~mm}$. A left vocal cord paralysis was confirmed and cervical computed tomography $(C T)$ showed multiple thyroid nodules of up to $35 \mathrm{~mm}$ associated with bilateral cervical lymph nodes (LN). Positron emission tomography $\left({ }^{18} \mathrm{~F}-F D G\right.$ PET/CT) evidenced hypermetabolic activity in bilateral cervical LN, lungs, pancreas and left intercostal soft tissue, as well as left gluteus. Thyroid biopsy reported a tall-cell variant of PTC, and endoscopic ultrasound guided fine needle aspiration (EUS-FNA) of pancreatic mass confirmed PTC metastasis. The molecular study was positive for BRAFV600E. Pancreatic metastasis from PTC can be accurately diagnosed with ${ }^{18} \mathrm{~F}-F D G$ PET/CT and EUS-FNA, which is consistent with a predominant expression of BRAFV600E mutation and, thus, an aggressive presentation with poor short-term survival.

(Rev Med Chile 2020; 148: 1025-1030)

Key words: Endoscopic Ultrasound-Guided Fine Needle Aspiration; Proto-Oncogene Proteins B-raf; Thyroid Cancer, Papillary.
'Departamento de

Endocrinología, Facultad de

Medicina, Pontificia Universidad

Católica de Chile. Santiago, Chile.

${ }^{2}$ Departamento de

Gastroenterología, Facultad de

Medicina, Pontificia Universidad

Católica de Chile. Santiago, Chile.

${ }^{3}$ Departamento de Cirugía de

Cabeza y Cuello, Facultad de

Medicina, Pontificia Universidad

Católica de Chile. Santiago, Chile.

${ }^{4}$ Departamento de Anatomía

Patológica, Facultad de Medicina,

Pontificia Universidad Católica de

Chile. Santiago, Chile.

${ }^{5}$ Centro Traslacional de

Endocrinología (CETREN)-UC.

Santiago, Chile.

aAlumna de pregrado, Pontificia

Universidad Católica de Chile,

Santiago de Chile.

Trabajo no recibió financiamiento. Los autores declaran no tener conflictos de interés.

Recibido el 16 de diciembre de 2019, aceptado el 3 de agosto de 2020.

Correspondencia a:

Dr. José Miguel Domínguez Ruiz-Tagle

Pontificia Universidad Católica de

Chile. Santiago, Chile.

Diagonal Paraguay 362, piso 4.

Santiago, Chile.

jdomingu@uc.cl
$\mathrm{E}$ 1 carcinoma papilar de tiroides (CPT) es el cáncer endocrinológico más frecuente. Las metástasis a distancia ocurren en 1-23\% de los pacientes, siendo pulmón y hueso los órganos frecuentemente comprometidos ${ }^{1,2}$. Rara vez se encuentra compromiso en otro órgano como cerebro, riñones, ovario, adrenales. Anecdóticamente, se han reportado casos de compromiso pancreático, especialmente debido al advenimiento de técnicas radiológicas como ${ }^{18} \mathrm{~F}-\mathrm{FDG} \mathrm{PET} / \mathrm{CT}^{3}$.

Si bien el CPT está generalmente asociado a un excelente pronóstico, cuando presenta metástasis a distancia la sobrevida a 5 años disminuye hasta 15-70\%, dependiendo del tamaño de las metástasis y su avidez por radioyodo ${ }^{1,4}$. La metástasis pancreática de CPT es extremadamente rara, reportándose solo 24 casos en la literatura ${ }^{5}$.

A continuación, presentamos un paciente que debutó con ictericia obstructiva secundaria a metástasis de CPT en cabeza de páncreas. Nuestro objetivo es ilustrar esta rara presentación y revisar sus características clínico-patológicas. 


\section{Caso clínico}

Hombre de 80 años que consultó por ictericia y disfonía de 3 meses de evolución. Al examen físico destacaba aumento de volumen pétreo cervical anterior, con adenopatías palpables en cadena yugular izquierda de consistencia similar. Del laboratorio destacó bilirrubina total 2,88 mg/dL $(<1,0)$, fosfatasas alcalinas $953 \mathrm{U} / \mathrm{L}(45-115)$, GGT $1.842 \mathrm{U} / \mathrm{L}(<60)$, VHS 57 (1-59), TSH 3,27 uU/ $\mathrm{mL}(0,3-4,2)$, tiroglobulina $165 \mathrm{ng} / \mathrm{mL}(3,5-77)$, anticuerpos anti-tiroglobulina $<1 \mathrm{Ul} / \mathrm{mL}(<4,11)$, calcitonina $<2 \mathrm{pg} / \mathrm{mL}(<18,2)$, antígeno carcinoembrionario 3ng/mL $(<3,8)$, CA19-9 26,9 U/ $\mathrm{mL}(<34)$.

La ecotomografía abdominal mostró dilatación de vía biliar y la resonancia magnética (RM) abdominal evidenció una masa de $36 \mathrm{~mm}$ en la cabeza pancreática, que comprimía el colédoco y dilataba la vía biliar proximal. La nasofibroscopía encontró parálisis de cuerda vocal derecha. La tomografía computada de cuello y tórax mostró múltiples nódulos tiroideos, el mayor de ellos en el lóbulo tiroideo izquierdo de $35 \mathrm{~mm}$, mal definido, de aspecto infiltrativo, adenopatías cervicales bilaterales e innumerables nódulos pulmonares de hasta $19 \mathrm{~mm}$, compatibles con localizaciones secundarias. El ${ }^{18} \mathrm{~F}$-FDG PET/CT mostró actividad hipermetabólica en las lesiones descritas, así como en partes blandas intercostal izquierda y músculo glúteo medio izquierdo (SUV max: 39,9) (Figura 1). La RM de cerebro mostró lesión nodular occipital derecha de $18 \mathrm{~mm}$, de carácter secundaria.

La punción aspirativa con aguja fina (PAF) del nódulo tiroideo izquierdo fue compatible con CPT (Bethesda VI). Se realizó PAF guiada por ultrasonido endoscópico (PAF-USE) de masa pancreática y se desobstruyó la vía biliar con una colangiopancreatografía retrógrada endoscópica (CPRE) e instalación de prótesis biliar metálica (Figura 1). El estudio citológico (Figura 2) informó células dispuestas en patrón papilar y en partes discohesivo con núcleos grandes, irregulares, vesiculosos, sobrepuestos con pliegues y pseudoinclusiones. El estudio inmunohistoquímico fue positivo para factor de transcripción tiroideo 1 (TTF-1) y tiroglobulina, y negativo para CEA, calcitonina, CD56 y CA19-9 (Figura 2). Estos hallazgos fueron compatibles con metástasis de CPT.

Se realizó tiroidectomía total con disección cervical central y lateral bilateral. El estudio histopatológico arrojó un CPT multifocal, variedad

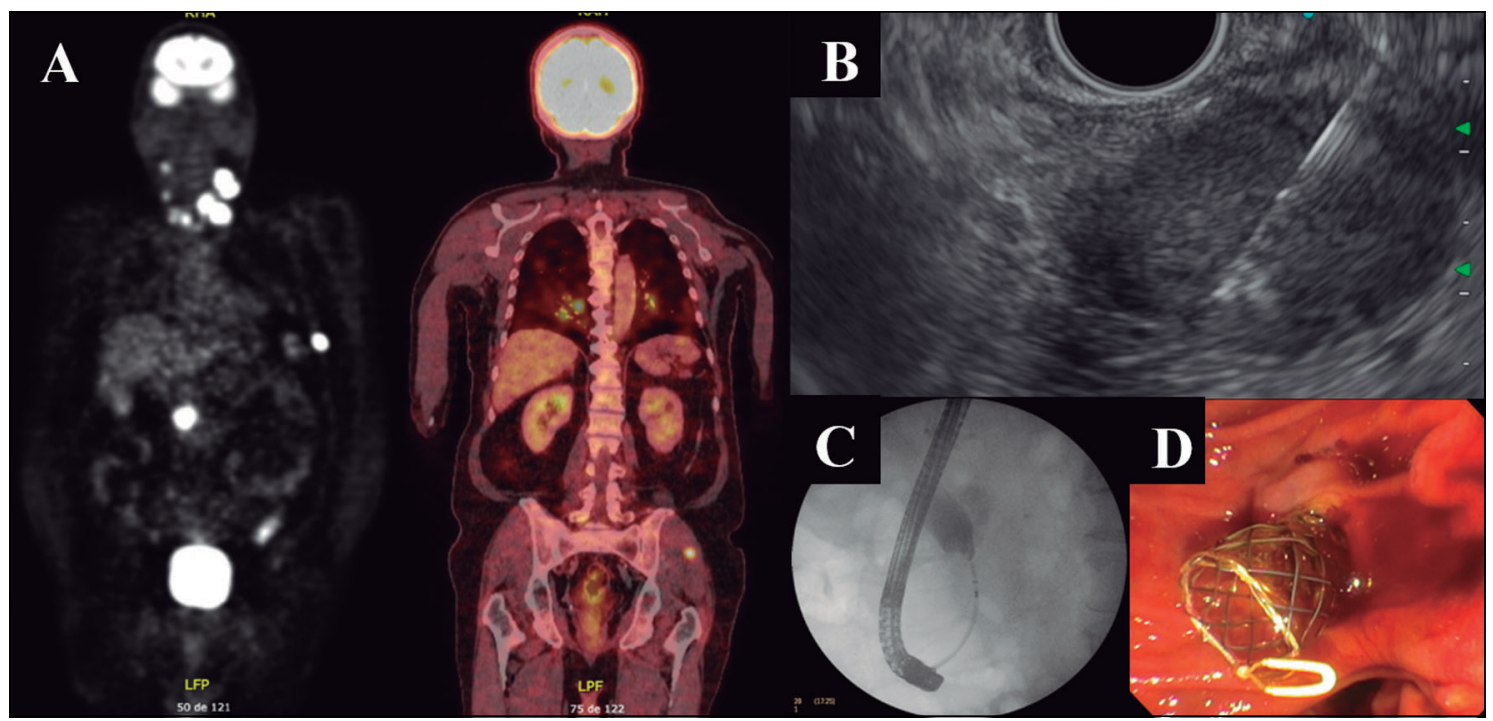

Figura 1. A: ${ }^{18} \mathrm{~F}-\mathrm{FDG}$ PET/CT actividad hipermetabólica en localizaciones: ganglionar cervical bilateral, pulmonar bilateral, pancreático y de partes blandas intercostal izquierda, así como en músculo glúteo medio ipsilateral. B: Endosonografía muestra lesión hipoecogénica a nivel de cabeza de páncreas de 39 × 28 mm. Punción con aguja vía endosonográfica para obtención de citología y block celular. C: Colangiopancreatografía retrógrada endoscópica (CPRE). D: Extremo de prótesis metálica instalada en vía biliar. 


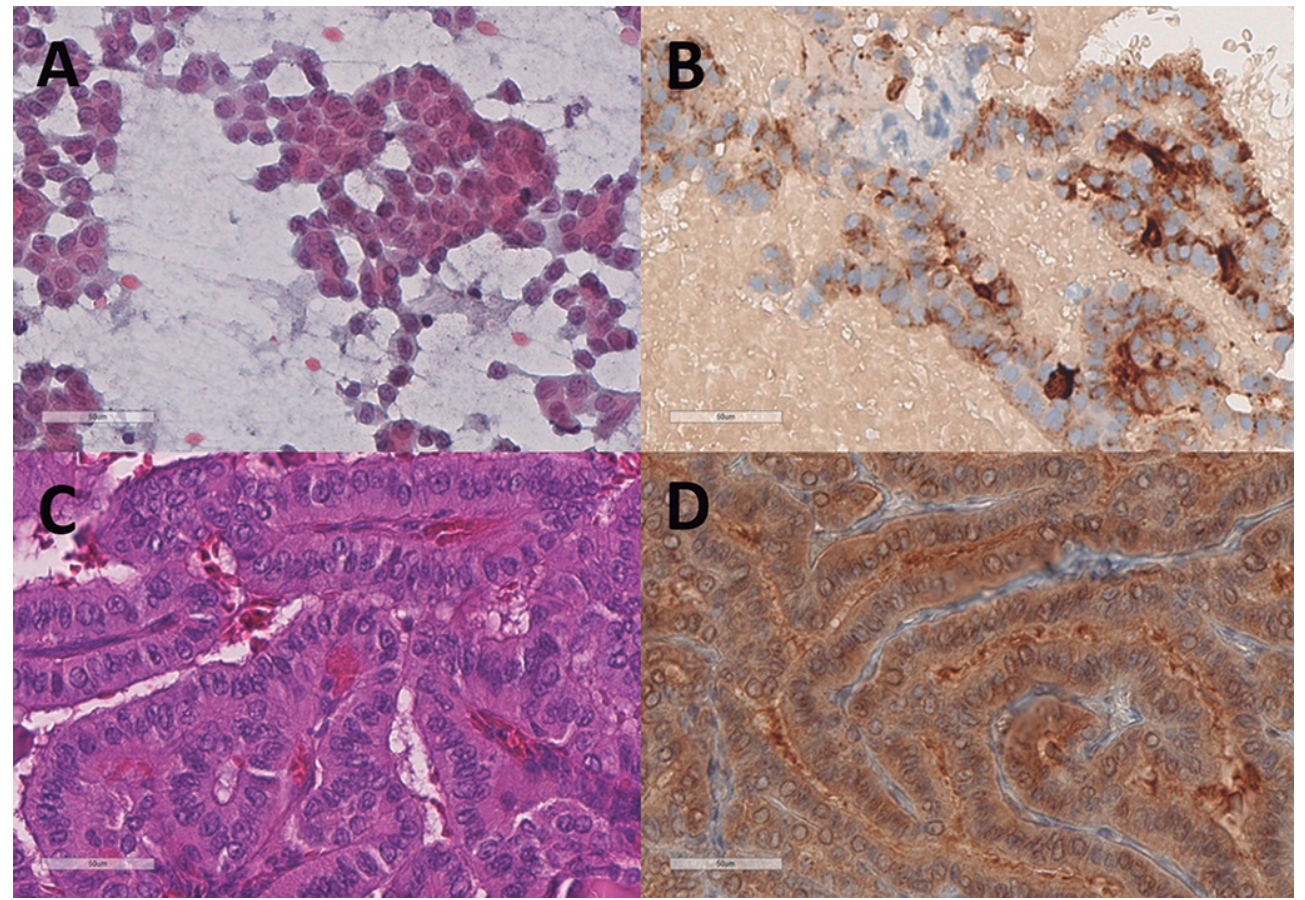

Figura 2. Metástasis de carcinoma papilar en páncreas: A: Extendido citológico en el que se observan grupos de células neoplásicas con estructuras papilares y núcleos grandes, irregulares, con pliegues y pseudoinclusiones (Papanicolau 40x). B: Estudio inmunohistoquímico demostró que las células neoplásicas son positivas para Tiroglobulina. C: Hallazgos microscópicos de la tiroidectomía. Se identificó un tumor con arquitectura papilar en la que se observó que las células son 3 veces más altas que anchas y sus núcleos son vesiculosos, con pliegues, sobrepuestos y con una que otra pseudoinclusión (Hematoxilina-eosina 40x). D: Estudio inmunohistoquímico de tiroidectomía positivo para BRAFV600E.

células altas (vCA), con foco de mayor tamaño $34 \mathrm{~mm}$, con extensión extratiroidea muscular, adiposa y bordes quirúrgicos extensamente positivos. Se observó infiltración perineural y vascular linfática, sin necrosis, ni componente anaplástico. Compromiso linfonodal metastásico extenso de hasta $28 \mathrm{~mm}$, con extensión extranodal.

Se complementó inmunohistoquímica mediante técnica automatizada en plataforma Benchmark Ultra de Ventana (Arizona, USA) con el anticuerpo anti-BRAF V600E (clon VE1), demostrando positividad en el tumor primario y metastásico (Figura 2). El estado mutacional fue confirmado utilizando el BRAF/NRAS Mutation Test (LSR) de ROCHE (RT-PCR), demostrándose mutación en codón 600 (V600E/E2/K) tanto en el tumor primario como en el metastásico.

Considerando los hallazgos descritos, se definió en equipo multidisciplinario manejo con radiocirugía de las lesiones pancreática y cerebral, junto con radioterapia externa cervical. Nueve meses post cirugía, las metástasis extracervicales se mantenían estables, por lo que se desestimó transitoriamente el uso de inhibidores de tirosin kinasa (ITK) y se administró radioyodo $200 \mathrm{mCi}$. La exploración sistémica con ${ }^{131} \mathrm{I}$ (ES- $\left.{ }^{131} \mathrm{I}\right)$ evidenció captación de yodo en el lecho tiroideo, sin captación en las lesiones extracervicales. Un año después de la primera cirugía, no ha evidenciado progresión de las lesiones cervicales ni extracervicales. El paciente otorgó consentimiento para la publicación de su caso clínico.

\section{Discusión}

Describimos el caso de un paciente que debutó con ictericia obstructiva por metástasis pancreática de CPT. Esta presentación agresiva es inusual y podría explicarse por características clínicas, 
subtipo histológico y alteraciones moleculares propias del tumor.

La metástasis de CPT en páncreas es extremadamente rara, con 24 casos publicados hasta la fecha, de edad promedio 59 años (rango 39-82). El tiempo transcurrido entre el diagnóstico inicial de CPT y la aparición de la metástasis pancreática fue 83 meses (rango 0-192), con aparición hasta 10 años luego de la presentación inicial ${ }^{5}$. Respecto al pronóstico, la sobrevida promedio reportada es de 8,7 meses, con 5 pacientes que han sobrevivido más de 4 años ${ }^{5,6}$.

En nuestro caso, el diagnóstico preoperatorio fue realizado con PAF-USE, similar a lo descrito en $8 / 24$ casos reportados, resultando ser una técnica precisa para el diagnóstico, que permite evitar la biopsia abierta $^{5,7}$. USE se reconoce como el examen más sensible (94-100\%) para detectar lesiones tumorales pancreáticas, junto con su capacidad de distinguir lesiones primarias de metastásicas y la realización de punción por aguja fina ${ }^{8,9}$. Entre las características endosonográficas de las metástasis pancreáticas se incluye la hipoecogenicidad, heterogeneidad y bordes variablemente definidos ${ }^{10}$. En la mayoría de los casos se presentan como lesión solitaria en la cabeza del páncreas ${ }^{5,10,11}$. La presencia de dilatación del conducto pancreático se ha reportado hasta en $20 \%$ de los casos ${ }^{10}$.

Tradicionalmente, la avidez de las células foliculares tiroideas por yodo radioactivo ha permitido el uso de la ES- ${ }^{131}$ I para el diagnóstico y seguimiento de las metástasis a distancia de CPT. Sin embargo, en el caso de la localización pancreática, su rendimiento es muy bajo. Davidson et al. estudiaron ES- ${ }^{131} \mathrm{I}$ en 7 casos, ninguno presentó avidez en páncreas ${ }^{12}$. La explicación podría estar en una disminución de la expresión génica del cotransportador $\mathrm{Na} / \mathrm{I}$ que caracteriza a los cánceres tiroideos menos diferenciados ${ }^{13}$.

El ${ }^{18} \mathrm{~F}-\mathrm{FDGPET} / \mathrm{CT}$ mostró hipermetabolismo en todas las lesiones del paciente, similar a otros 12 casos en que hubo aumento anormal del trazador, lo que es un signo de desdiferenciación tumoral y un marcador de agresividad y mal pronóstico ${ }^{5,14,15}$. Molecularmente, estos tumores se caracterizan por una mayor expresión del transportador GLUT-1, que capta glucosa, y una reducción de todos los genes asociados a diferenciación tiroidea, entre los que destaca el NIS, que codifica para el cotransportador $\mathrm{Na} / \mathrm{I}^{13}$.

Si bien la variedad usual es la forma de CPT más reportada en metástasis pancreáticas, nuestro caso se diferencia por tratarse de un CPT-vCA. Este es el cuarto reporte de CPT-vCA con metástasis pancreática descrito en la literatura, y comparte con los demás una presentación agresiva, avidez por ${ }^{18} \mathrm{~F}-\mathrm{FDG}$, ausencia de captación de ${ }^{131} \mathrm{I} y$, cuando se realizó estudio molecular, mutación BRAFV600E positivo $^{7,12,14}$. Esto confirma el curso agresivo de estos tumores, asociado a mayor incidencia de metástasis a distancia, recurrencia y mortalidad, con una sobrevida global y libre de enfermedad a 10 años menor que la variedad usual ${ }^{4}$.

La inmunohistoquímica es una herramienta útil para definir el origen tumoral en lesiones sospechosas de metástasis. En nuestro caso, la positividad para TTF-1 y tiroglobulina confirmaron el origen tiroideo. A pesar de la presentación clínica agresiva, llama la atención la ausencia de signos de desdiferenciación (necrosis y mitosis) en el tumor primario y metástasis.

Algunas mutaciones driver como de los genes $R A S, R E T / P T C$ y $B R A F$ son relevantes para la carcinogénesis del CPT. La mutación BRAFV600E ha sido descrita en $45-50 \%$ de los casos y está asociada a formas agresivas de CDT, mayor avidez a ${ }^{18} \mathrm{~F}$-FDGPET/CT y menor captación de ${ }^{131} \mathrm{I}$ en las metástasis a distancia ${ }^{13,16,17}$. Esta mutación aumenta la producción de moléculas promotoras de cáncer como proteínas de matriz extracelular y factor de crecimiento derivado de plaqueta a través de la activación constitutiva de la vía MAPK ${ }^{15}$. BRAFV600E ha sido estudiada solo en 6 casos de metástasis pancreática además del nuestro, siendo positivo en $85 \%$ (Tabla 1$)^{5,12,18-20}$.

En relación a la terapia, por tratarse de CPT con las características descritas, es planteable el uso de ITK en caso de progresión clínicamente significativa. La evidencia de ITK en metástasis pancreáticas de CPT es escasa. Se ha reportado el uso de sorafenib en dos casos, con respuesta parcial en uno y progresión en el otro ${ }^{19,20}$. En nuestro paciente, dada la estabilidad de las lesiones extracervicales, no se ha indicado ITK. Sin embargo, es una alternativa por considerar de acuerdo a la evolución clínica.

En suma, nuestro paciente y los casos previamente descritos muestran que los pacientes con metástasis pancreática de CPT pueden ser diagnosticados de manera certera con ${ }^{18} \mathrm{~F}$-FDG PET/CT y PAF-USE. Se caracterizan por escasa avidez y respuesta a radioyodo, con presencia de 
Tabla 1. Casos de metástasis pancreática secundaria a carcinoma papilar de tiroides con estudio genético

\begin{tabular}{|c|c|c|c|c|c|c|c|c|c|c|}
\hline $\begin{array}{l}\text { Refe- } \\
\text { rencia }\end{array}$ & Autor & $\begin{array}{c}\text { Edad/ } \\
\text { Género }\end{array}$ & $\begin{array}{l}\text { Tipo } \\
\text { tumor }\end{array}$ & $\begin{array}{l}\text { Otras loca- } \\
\text { lizaciones } \\
\text { metastásicas }\end{array}$ & $\begin{array}{l}{ }^{18} \text { F-FDG } \\
\text { PET/CT }\end{array}$ & $\begin{array}{c}\text { Avidez } \\
\left.\right|^{-131}\end{array}$ & $\begin{array}{c}\text { Diag- } \\
\text { nóstico }\end{array}$ & $\begin{array}{c}\text { Test } \\
\text { genético }\end{array}$ & $\begin{array}{l}\text { Trata- } \\
\text { miento }\end{array}$ & $\begin{array}{l}\text { Segui- } \\
\text { miento }\end{array}$ \\
\hline 18 & Borschitz & $\begin{array}{c}46 \text { / } \\
\text { Hombre }\end{array}$ & CPT & Pulmón, hueso & Ávido & No & $\begin{array}{c}\text { TC, } \\
\text { 18F-FDG } \\
\text { PET/CT }\end{array}$ & $\begin{array}{l}\text { Positivo } \\
\text { BRAF } \\
\text { V600E }\end{array}$ & Cirugía & Progresión \\
\hline 18 & Borschitz & $\begin{array}{c}34 \text { / } \\
\text { Mujer }\end{array}$ & CPT-VF & N/A & Ávido & No & $\begin{array}{c}\text { TC, } \\
\text { 18F-FDG } \\
\text { PET/CT }\end{array}$ & $\begin{array}{c}\text { Negativo } \\
\text { BRAFV } \\
600 E \\
\text { RET/PTC1 }\end{array}$ & Cirugía & N/A \\
\hline 19 & Tunio & $\begin{array}{c}56 / \\
\text { Mujer }\end{array}$ & CPT-vCA & Pulmón & Ávido & No & $\begin{array}{l}\text { TC, } \\
\text { RM }\end{array}$ & $\begin{array}{l}\text { Positivo } \\
\text { BRAF } \\
\text { V600E }\end{array}$ & $\begin{array}{c}\text { Cirugía, } \\
\text { sorafenib }\end{array}$ & $\begin{array}{c}\text { Respuesta } \\
\text { parcial }\end{array}$ \\
\hline 20 & Alzahrani & $\begin{array}{c}55 \text { / } \\
\text { Hombre }\end{array}$ & CPTC & $\begin{array}{l}\text { Pulmón, } \\
\text { hueso, hígado, } \\
\text { omento }\end{array}$ & Ávido & No & $\begin{array}{l}\text { RM, } \\
\text { USE }\end{array}$ & $\begin{array}{l}\text { Positivo } \\
\text { BRAF } \\
\text { V600E }\end{array}$ & Sorafenib & Progresión \\
\hline 6 & Murakami & $\begin{array}{c}80 \text { / } \\
\text { Mujer }\end{array}$ & CPT & Pulmón & Ávido & No & $\begin{array}{c}\text { TC, } \\
\text { 18F-FDG } \\
\text { PET/CT, } \\
\text { RM, } \\
\text { USE }\end{array}$ & $\begin{array}{l}\text { Positivo } \\
\text { BRAF } \\
\text { V600E }\end{array}$ & Cirugía & Progresión \\
\hline \multirow[t]{2}{*}{12} & Davidson & $\begin{array}{c}84 \text { / } \\
\text { Mujer }\end{array}$ & CPT-vCA & N/A & Ávido & No & $\begin{array}{c}\text { TC, } \\
\text { 18F-FDG } \\
\text { PET/CT, } \\
\text { USE }\end{array}$ & $\begin{array}{l}\text { Positivo } \\
\text { BRAF } \\
\text { V600E }\end{array}$ & $\begin{array}{l}\text { Segui- } \\
\text { miento }\end{array}$ & Progresión \\
\hline & $\begin{array}{c}\text { Caso } \\
\text { presente }\end{array}$ & $\begin{array}{c}80 / \\
\text { Hombre }\end{array}$ & CPT-vCA & $\begin{array}{l}\text { Pulmón, } \\
\text { cerebro, } \\
\text { músculo }\end{array}$ & Ávido & N/A & $\begin{array}{c}\text { TC, } \\
\text { 18F-FDG } \\
\text { PET/CT, } \\
\text { RM, } \\
\text { USE }\end{array}$ & $\begin{array}{l}\text { Positivo } \\
\text { BRAF } \\
\text { V600E }\end{array}$ & N/A & N/A \\
\hline
\end{tabular}

CPT: carcinoma papilar de tiroides; CPT VF: carcinoma papilar de tiroides variedad folicular; CPT vCA: carcinoma papilar de tiroides variedad células altas; CPT c: carcinoma papilar de tiroides variedad clásica; TC: tomografía computada; RM: resonancia magnética; USE: ultrasonografía endoscópica.

mutación BRAFV600E, lo que se asocia a una pobre sobrevida. La terapia multimodal con cirugía, radioterapia y eventual uso de ITK podría mejorar el pronóstico de estos pacientes.

Agradecimientos: Claudio Pinto Viguera por procesar y fotografiar las muestras de anatomía patológica.

\section{Referencias}

1. Goffredo P, Sosa JA, Roman SA. Differentiated thyroid cancer presenting with distant metastases: a population analysis over two decades. World J Surg 2013; 37 (7): 1599-605. doi: 10.1007/s00268-013-2006-9. PubMed PMID: 23525600.

2. Dinneen SF, Valimaki MJ, Bergstralh EJ, Goellner JR, Gorman CA, Hay ID. Distant metastases in papillary thyroid carcinoma: 100 cases observed at one institution during 5 decades. J Clin Endocrinol Metab 1995; 80 (7): 2041-5. doi: 10.1210/jcem.80.7.7608252. PubMed PMID: 7608252.

3. Angeles-Angeles A, Chable-Montero F, MartíinezBenítez B, Albores-Saavedra J. Unusual metastases of papillary thyroid carcinoma: report of 2 cases. Ann Diagn Pathol 2009; 13 (3): 189-96. Epub 2008/06/26. 
doi: 10.1016/j.anndiagpath.2008.02.010. PubMed PMID: 19433299.

4. Wang LY, Palmer FL, Nixon IJ, Thomas D, Patel SG, Shaha AR, et al. Multi-organ distant metastases confer worse disease-specific survival in differentiated thyroid cancer. Thyroid 2014; 24 (11): 1594-9. Epub 2014/09/24. doi: 10.1089/thy.2014.0173. PubMed PMID: 25162180.

5. Murakami Y, Shimura T, Okada R, Kofunato Y, Ishigame T, Yashima R, et al. Pancreatic metastasis of papillary thyroid carcinoma preoperatively diagnosed by endoscopic ultrasound-guided fine-needle aspiration biopsy: a case report with review of literatures. Clin J Gastroenterol 2018; 11 (6): 521-9. Epub 2018/06/08. doi: 10.1007/s12328-018-0875-z. PubMed PMID: 29948817.

6. Meyer A, Behrend M. Is pancreatic resection justified for metastasis of papillary thyroid cancer? Anticancer Res 2006; 26 (3B): 2269-73. PubMed PMID: 16821600.

7. Siddiqui AA, Olansky L, Sawh RN, Tierney WM. Pancreatic metastasis of tall cell variant of papillary thyroid carcinoma: diagnosis by endoscopic ultrasound-guided fine needle aspiration. JOP 2006; 7 (4): 417-22. Epub 2006/07/10. PubMed PMID: 16832140.

8. Sekulic M, Amin K, Mettler T, Miller LK, Mallery S, Stewart J. Pancreatic involvement by metastasizing neoplasms as determined by endoscopic ultrasound-guided fine needle aspiration: A clinicopathologic characterization. Diagn Cytopathol 2017; 45 (5): 418-25. Epub 2017/02/16. doi: 10.1002/dc.23688. PubMed PMID: 28205397.

9. Gress FG, Hawes RH, Savides TJ, Ikenberry SO, Cummings $\mathrm{O}$, Kopecky K, et al. Role of EUS in the preoperative staging of pancreatic cancer: a large single-center experience. Gastrointest Endosc 1999; 50 (6): 786-91. doi: 10.1016/s0016-5107(99)70159-8. PubMed PMID: 10570337.

10. Atiq M, Bhutani MS, Ross WA, Raju GS, Gong Y, Tamm $\mathrm{EP}$, et al. Role of endoscopic ultrasonography in evaluation of metastatic lesions to the pancreas: a tertiary cancer center experience. Pancreas 2013; 42 (3): 516-23. doi: 10.1097/MPA.0b013e31826c276d. PubMed PMID: 23211369.

11. Palazzo L, Borotto E, Cellier C, Roseau G, Chaussade $\mathrm{S}$, Couturier D, et al. Endosonographic features of pancreatic metastases. Gastrointest Endosc 1996; 44 (4): 433-6. doi: 10.1016/s0016-5107(96)70095-0. PubMed PMID: 8905364.

12. Davidson M, Olsen RJ, Ewton AA, Robbins RJ. Pancreas metastases from papillary thyroid carcinoma: a review of the literature. Endocr Pract 2017; 23 (12): 1425-9. Epub 2017/11/16. doi: 10.4158/EP-2017-0001. PubMed PMID: 29144798.

13. Yang $\mathrm{K}$, Wang $\mathrm{H}$, Liang Z, Liang J, Li F, Lin Y. BRAF$\mathrm{V} 600 \mathrm{E}$ mutation associated with non-radioiodine-avid status in distant metastatic papillary thyroid carcinoma. Clin Nucl Med 2014; 39 (8): 675-9. doi: 10.1097/ RLU.0000000000000498. PubMed PMID: 24978326.

14. Jobran R, Baloch ZW, Aviles V, Rosato EF, Schwartz S, LiVolsi VA. Tall cell papillary carcinoma of the thyroid: metastatic to the pancreas. Thyroid 2000; 10 (2): 185-7. doi: 10.1089/thy.2000.10.185. PubMed PMID: 10718557.

15. Kim TH, Park YJ, Lim JA, Ahn HY, Lee EK, Lee YJ, et al. The association of the BRAF(V600E) mutation with prognostic factors and poor clinical outcome in papillary thyroid cancer: a meta-analysis. Cancer 2012; 118 (7): 1764-73. Epub 2011/08/31. doi: 10.1002/cncr.26500. PubMed PMID: 21882184.

16. Tang KT, Lee $\mathrm{CH}$. BRAF mutation in papillary thyroid carcinoma: pathogenic role and clinical implications. J Chin Med Assoc 2010; 73 (3): 113-28. doi: 10.1016/ S1726-4901(10)70025-3. PubMed PMID: 20230995.

17. Santhanam P, Khthir R, Solnes LB, Ladenson PW. The relationship of BRAF. Endocr Pract 2018; 24 (1): 21-6. Epub 2017/11/16. doi: 10.4158/EP-2017-0080. PubMed PMID: 29144823.

18. Borschitz T, Eichhorn W, Fottner C, Hansen T, Schad A, Schadmand-Fischer S, et al. Diagnosis and treatment of pancreatic metastases of a papillary thyroid carcinoma. Thyroid 2010; 20 (1): 93-8. doi: 10.1089/thy.2009.0026. PubMed PMID: 20025539; PubMed Central PMCID: PMCPMC2833176.

19. Tunio MA, Alasiri M, Riaz K, Alshakweer W. Pancreas as delayed site of metastasis from papillary thyroid carcinoma. Case Rep Gastrointest Med 2013; 2013: 386263. Epub 2013/03/27. doi: 10.1155/2013/386263. PubMed PMID: 23607002; PubMed Central PMCID: PMCPMC3623391.

20. Alzahrani AS, AlQaraawi A, Al Sohaibani F, Almanea $\mathrm{H}$, Abalkhail H. Pancreatic metastasis arising from a BRAF(V600E)-positive papillary thyroid cancer: the role of endoscopic ultrasound-guided biopsy and response to sorafenib therapy. Thyroid 2012; 22 (5): 536-41. Epub 2012/03/21. doi: 10.1089/thy.2011.0247. PubMed PMID: 22435913. 